\title{
Tandem Mass Spectrometry on a Miniaturized Laser Desorption Time-of-Flight Mass Spectrometer
}

\author{
Xiang Li \\ University of Maryland, \\ Baltimore County \\ Baltimore, MD 21250 \\ 301-614-6016 \\ xiang.li@nasa.gov
}

\author{
Timothy Cornish, \\ Ecelberger C\&E Research, Inc. \\ Catonsville, MD 21228 \\ 301-614-6016 \\ tcornish@CEResearchInc.com \\ SAE@CEResearchlnc.com
}

\author{
Scott Stephanie A. Getty, William B. \\ Brinckerhoff \\ NASA Goddard Space Flight \\ Center \\ Greenbelt, MD 20771 \\ 301-614-6397 \\ stephanie.a.getty@nasa.gov
}

\begin{abstract}
Tandem mass spectrometry (MS/MS) is a powerful and widely-used technique for identifying the molecular structure of organic constituents of a complex sample. Application of MS/MS to the study of unknown planetary samples on a remote space mission would contribute to our understanding of the origin, evolution, and distribution of extraterrestrial organics in our solar system. Here we report on the realization of MS/MS on a miniaturized laser desorption time-of-flight mass spectrometer (LD-TOF-MS), which is one of the most promising instrument types for future planetary missions. This achievement relies on two critical components: a curved-field reflectron and a pulsedpin ion gate. These enable use of the complementary post-source decay (PSD) and laser-assisted collision induced dissociation (L$\mathrm{CID}) \mathrm{MS} / \mathrm{MS}$ methods on diverse measurement targets with only modest investment in instrument resources such as volume and weight. MS/MS spectra of selected molecular targets in various organic standards exhibit excellent agreement when compared with results from a commercial, laboratory-scale TOF instrument, demonstrating the potential of this powerful technique in space and planetary environments.
\end{abstract}

Index Terms - laser desorption time-of-flight mass spectrometer (LD-TOF-MS), laser desorption mass spectrometry (LDMS), tandem mass spectrometry (MS/MS), post-source decay (PSD), laser-assisted collision induced dissociation (L-CID)

\section{TABLE OF CONTENTS}

1.INTRODUCTION 1

2. INSTRUMENT DESIGN. 23.

EXPERIMENTAL METHODS 3

4. RESULTS. 4

5. DISCUSSION...

6.CONCLUSIONS

REFERENCES. .7

BIOGRAPHY. 7

\section{INTRODUCTION}

Mass spectrometers (MS) have been employed on a number of space missions to measure the atomic, molecular, and william.b.brinckerhoff@nasa.gov isotopic composition of remote planetary environments (1). Such studies have informed our understanding of the origin and evolution of our solar system, the origin of life on Earth, and the potential for life elsewhere. Many of the targets prioritized for future planetary exploration, such as comets, asteroids, and planetary satellites, are characterized by great chemical complexity, requiring advances in remote molecular analysis technique. In tandem mass spectrometry (also called MS/MS, for "mass spectrometry/mass spectrometry"), a particular ion or range of ions is selected from an initial mass spectrum, isolated by removing all other species from further analysis, and subsequently fragmented (most commonly through collisions with gas). The resulting fragmentation pattern is thus associated exclusively with the original ion/s, enabling high-confidence identification and structural analysis. MS/MS is compatible with a variety of mass analyzers used in space flight. For example, the linear ion trap mass spectrometer in the Mars Organic Molecule Analyzer (MOMA) on the ExoMars rover $(2,3)$ uses MS/MS to isolate and fragment molecular ions of interest, to address mission requirements to detect potential molecular biosignatures in Mars surface and subsurface materials.

The laser desorption time-of-flight mass spectrometer (LDTOF-MS) is widely employed in analytical laboratories due to its straightforward operation, high-mass detection, high sensitivity, and rapid analysis times. While such instruments are large, often containing ion flight tubes over one meter long to maximize resolution, LD-TOF-MS is particularly well suited to miniaturization and remote application due to its lightweight design, simple electronics (e.g., no RF power supplies are required), and direct compatibility with airless environments. In previous work, we have demonstrated the powerful analytical capabilities of a compact LD-TOF-MS for space missions (4). Adapting the MS/MS capability to a miniaturized TOF mass spectrometer has required development of specialized approaches beyond those employed on commercial instrumentation. For example, MS/MS on the Bruker Autoflex Speed MALDI TOF/TOF (5) employs a separate high-voltage "LIFT" collision cell to fragment and reaccelerate product ions. This approach would add substantial weight/volume, power consumption, and additional mechanisms to a miniature instrument. In this study, we describe our MS/MS approach, which combines a curved field reflectron and a pulsed pin ion 
gating technique. Fragmentation is accomplished by spontaneous (passive) post-source decay (PSD) or by laserinduced (active) collision induced dissociation (L-CID). In this setup, minimal additional weight/volume is required. The usual external supply of collision gas is not needed, rendering the instrument far more suitable for space missions. To demonstrate the performance of this approach, the MS/MS spectra of selected peaks in various space-relevant organic standards were measured and compared to commercial TOF instrument results.

\section{INSTRUMENT DESIGN}

2.1 Tandem Mass Spectrometry (MS/MS)

The miniaturized LD-TOF-MS has been previously reported (4). Briefly, the instrument prototype uses low voltages $(<5 \mathrm{kV})$, measures less than $30 \mathrm{~cm}$ long, and weighs $\sim 5 \mathrm{~kg}$ including laser and all electronics (4). A key component of the mass spectrometer is the cylindrical, gridless curved-field reflectron (CFR) that has been described elsewhere $(6,7)$. The enhanced instrument also features a pulsed pin ion gate to isolate a targeted ion peak at a specific time. Both features are described below. In the simplest LD-TOF-MS configuration, a single UV laser (355 nm from a Nd:YAG laser, or $337 \mathrm{~nm}$ from a $\mathrm{N}_{2}$ laser) is focused onto the surface of a solid sample to generate both positive and negative ions within a few nanosecond pulse width. The simple mass analyzer provides unit mass resolution at several hundred Da (4). To achieve collision induced dissociation (CID) in MS/MS mode, a second laser beam is directed onto a solid surface along the path of drifting ion beam, creating a highly-localized plume of neutrals and ions to collide with (and thereby fragment) the ions of interest.

The Curved Field Reflectron (CFR): The curvature of the potential gradient in the CFR is used to focus ions of widely varying kinetic energies entering the reflector. To illustrate the versatility of its operation, consider two types of fragment ions. Stable (prompt) fragment ions formed at the ion source acquire the full translational energy of source voltage, penetrate the full depth of the reflector, and are brought into focus at the detector surface. This is the basic operational principle of a standard reflectron TOF-MS. In comparison, product ions formed subsequent to their exit from the source (so called post source decay (PSD) ions), have reduced translational energy due to their loss of mass during the fragmentation process in the field-free drift region. Hence these ions penetrate less into the reflectron and would thus arrive at the detector entirely unfocused in a conventional linear gradient reflectron. In the CFR analyzer, however, these reduced energy ions are focused at the same detector plane as the full energy ions formed in the ion source (Figure 1). Where traditional TOF reflector analyzers are capable of resolving stable fragmented ions, the CFR analyzer focuses both stable and PSD ions simultaneously. This capability adds significant benefit in MS/MS analysis mode where both

precursor and PSD ions can be detected simultaneously. While this feature has major implications for recovering and deconvolution of PSD product ion spectra, the challenge is to isolate and identify these product ions and formulate a precise calibration scale so that this is a powerful analytical feature rather than one that merely complicates the stable ion spectrum.

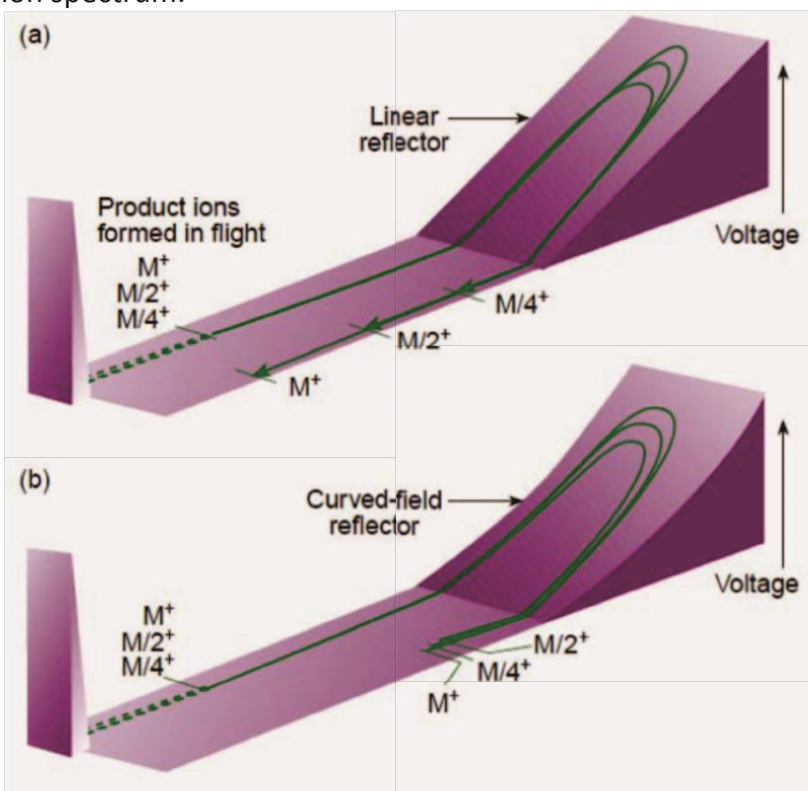

Figure 1. Comparison of the focusing properties between the (a)standard linear reflector and the (b)curved-field reflector. The focal points of the product ions are widely separated from each other in the case of linear reflector while they coalesce to a single point in the case of curved-field reflector.

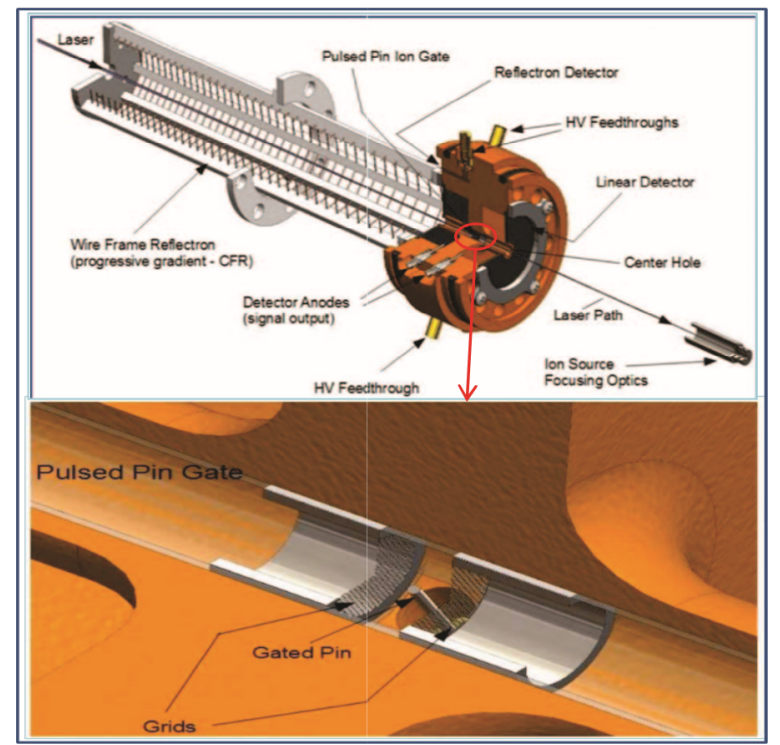

Figure 2. (Top) The detailed wire fame reflector and integrated detector body. (Bottom) Detail of the pulsed-pin ion gate.

Pulsed-Pin Ion gate: Ion gates have been used for decades to separate ion clusters in a 
beam. This is a particularly useful device to develop the tandem TOF-MS. In our case, the gate consists of a single thin wire that extends between two grids held at the drift potential (Figure 2). The wire is pulsed to $1.5 \mathrm{kV}$ relative to the drift potential in order to deflect those ions passing through the gate region so that they are suppressed from detection. lons of interest pass through the gate only during the period in which the wire is returned to the drift potential.

Post Source Decay (PSD): A highly fortuitous property of product ion formation in a TOF mass analyzer is that PSD products, formed within the drift region, travel in a cluster along with the ions from which they are formed (precursor ions) at the same velocity and pass through the ion gate at precisely the same time. Therefore, if the gate is pulsed "low" (off) to allow a particular targeted mass through (at a specific time), both precursor and product ions associated with that precursor pass through together, while all other ions are eliminated from that spectrum using the deflection properties of the gate. As mentioned earlier, the PSD ions would be detected along with parent ions by the CFR mass analyzer, therefore forming a MS/MS spectrum. Detection of PSD ions has been found to be very useful in biochemical identification, particularly for the sequencing information it contributes in peptide analyses.

Laser-Assisted Collision Induced Dissociation (L-CID): Traditional CID employs a neutral collision gas (such as helium or argon) to promote dissociation through direct collisions of the injected gas with the ion beam. Highly limited gas storage, regulation, pumping capabilities in flight instruments render gas cell CID an less-desirable option in our miniaturized LDTOF-MS. We therefore devised a relatively simple method to implement the MS/MS feature by adding a second laser beam precisely synchronized to the main desorption/ionization laser pulse to deliver a short burst of collision gas into the path of the ion beam (Laser-Assisted CID). Since the gas plume generation and expansion is very slow compared to the time scale of ion beam passage, no better timing control other than synchronizing the two lasers is needed, i.e., all the ions generated from the ion source would pass through the plume for collisions to occur. Using this method, negligible additional pressure is generated by the L-CID process, thereby eliminating the need for increased pumping capability. Compared to the ion trap's low-energy collision processes, L$\mathrm{CID}$ in the TOF-MS ion flight path are high-energy events $(>5000 \mathrm{~V})$, a feature that is often favorable for unique product identification. We expect to measure most MS/MS spectra through gating and PSD detection, but L-CID would add value for specific target compounds that require higher collision

energy for unique MS/MS characterization.

\subsection{Benefits of MS/MS}

In $\mathrm{MS} / \mathrm{MS}$, individual molecular species can be separated from a complex background, allowing for detection and assignment of its product ions. Additionally, when lower mass matrix or salt ions are ejected from the ion beam, the ions of interest (which pass unperturbed through the gate) can be detected with greater sensitivity and accuracy due to the elimination of microchannel plate (MCP) detector deadtime effects. Once a MCP channel is used to detect an ion, it requires about $1 \mathrm{~ms}$ for that channel to recharge in order to detect another ion, which is much longer than the total spectrum acquisition time. Moreover, since precursor and product ions pass through the gate simultaneously, it is found to be unnecessary for the parent ion to be detected in a product ion spectrum. That is, if the gate is tuned to allow passage of ions at a specified predetermined time, a characteristic product ion spectrum will identify the presence of the molecular species in the sample regardless of the fact that the intact molecular ion may remain undetected. This is a highly beneficial feature of tandem mass spectrometry in detecting those species that are not sufficiently stable to remain intact throughout the passage through the mass analyzer. Important analytes with reduced stability would not be observed in conventional MS analysis but would be detected through its product ion spectrum in a tandem instrument.

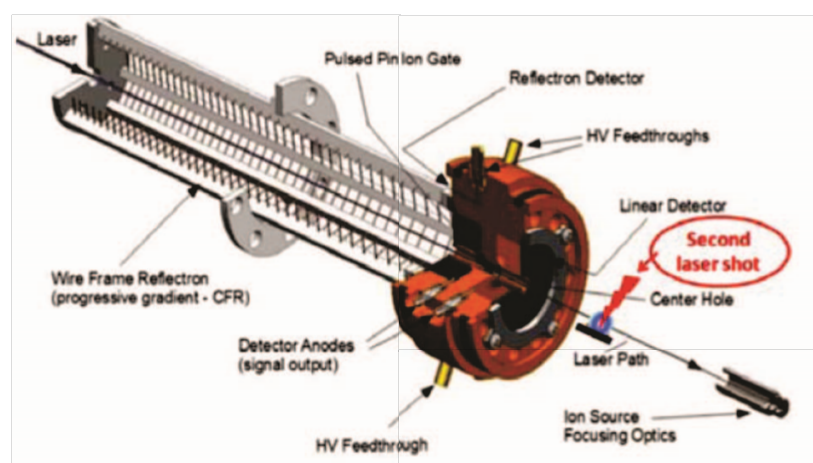

Figure 3. Schematic of Laser-assisted Collision Induced Dissociation (L-CID) setup in the LD-TOF-MS instrument.

\section{EXPERIMENTAL MethOdS}

In this study, we present MS/MS spectra of several representative samples, including D-cyano

-4-hydroxycinnamic acid (CHCA), tributylphosphate (TBP), the amino acid valine, two dipeptides (glycine-glycine and aspartame), and rhodamine 6G (R6G). CHCA itself is a commonly used Matrix Assisted Laser Desorption/Ionization (MALDI) matrix compound, and in the cases of valine, glycine-glycine, aspartame, and tributylphosphate samples, CHCA were deposited first on the sample stubs before the sample was deposited on top providing the MALDI effect in the ion generation. CHCA was solvated in 50:50 (0.1\% TFA in water):acetonitrile $(\mathrm{v} / \mathrm{v})$, and samples were either dissolved

or formed into a slurry in the same solution. R6G in methanol was dropped directly on the sample stub without matrix. All samples were dried in air, then transferred into the TOF-MS for pump-down prior to measurement. To desorb and ionize the sample, the UV laser energy was varied from 40 to $140 \mu \mathrm{J}$, corresponding to a range of peak power density between 77 and $450 \mathrm{MW} / \mathrm{cm}^{2}$. For the L-CID mode, a second nitrogen 
laser $(337 \mathrm{~nm})$ was used to ablate a graphite surface generating a local neutral plume to collide with the ion beam. Spectra can be obtained in both positive and negative ion modes, but we focused on the positive mode in this study.

To evaluate the performance of the prototype mass spectrometer, we also analyzed the same suite of samples utilizing a commercial MALDI TOF mass spectrometer (Bruker Autoflex Speed). In this instrument, samples were introduced into the instrument on a stainless steel plate with $\sim 2 \mathrm{uL}$ sample solution or slurries dried in air before transferring into the high vacuum environment. The ion source of the commercial instrument is equipped with a Nd:YAG laser (355 $\mathrm{nm},<5 \mathrm{~ns}$ pulse) focused to an elliptical spot with approximate dimensions of $0.2 \times 0.2 \mathrm{~mm}$. When operating in MS/MS mode, choices between PSD mode and CID (using argon) modes can be made, and in many cases the results showed negligible difference between the two modes.

\section{RESULTS}

Mass spectral data from each sample were compared between the miniaturized LD-TOF-MS and the commercial Bruker MALDI TOF-MS.

CHCA: The full mass spectrum of CHCA and the MS/MS spectrum of the selected peak, protonated dimer of $\mathrm{CHCA}$, at $\mathrm{m} / \mathrm{z} 379$ are shown in Figure 4. The full spectrum of CHCA is shown here to demonstrate that the pin gate can effectively select the target ion and the PSD ions along with it. The ion gating works similarly for all the samples tested here, so only MS/MS spectra for other samples are shown. When comparing the MS/MS spectrum of CHCA dimer obtained on the miniaturized TOF-MS to the corresponding spectrum on the Bruker commercial instrument, it is clear that the results are consistent with each other. The two major fragments of the CHCA dimer can be assigned as the protonated CHCA monomer and the monomer with the loss of an $-\mathrm{OH}$ group. Note that the CID operational mode on the commercial instrument did not enhance the product ion signal observed in the PSD spectrum.

TBP: Tributylphosphate is used as a solvent for extraction and purification of rare earth metals from their ores. This compound has three side chains bound to a central phosphate group. The MS/MS spectrum of the selected peak, protonated monomer of TBP $(\mathrm{m} / \mathrm{z} 267)$, is compared between the miniature LD-TOF-MS and commercial instrument (Figure

5). The results on both instruments are consistent showing

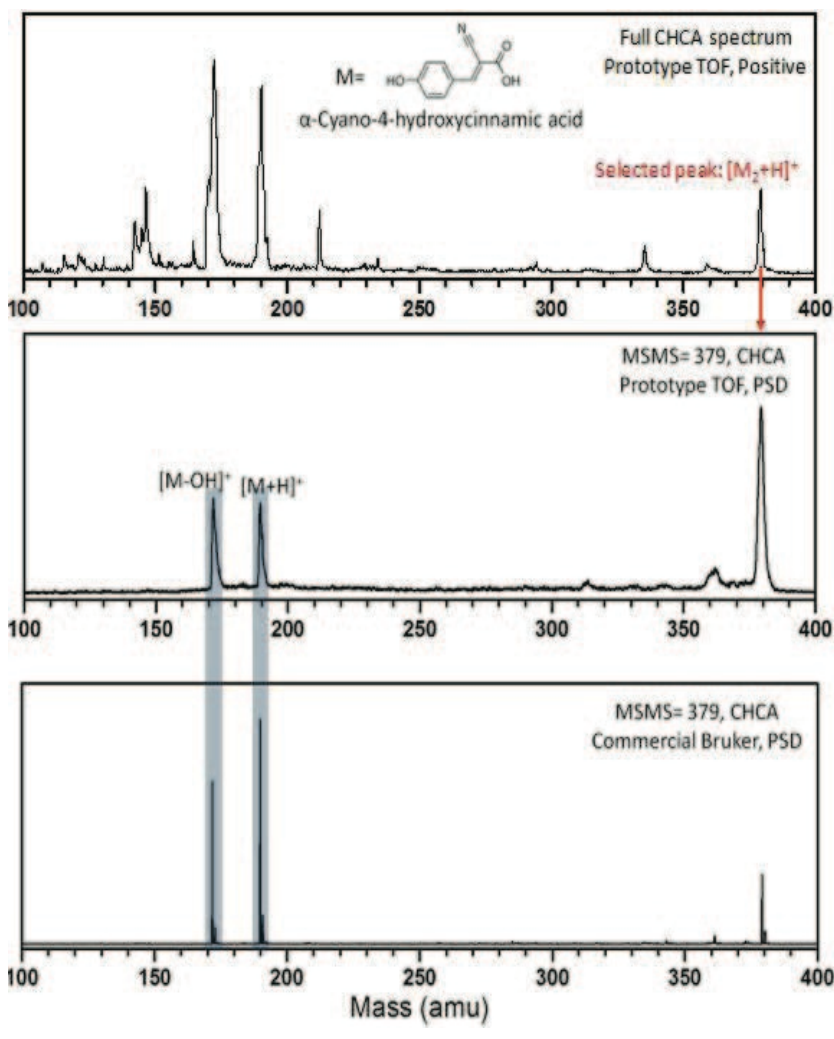

Figure 4. (TOP) Full mass spectrum of CHCA. (MIDDLE)

MS/MS spectrum of $\mathrm{m} / \mathrm{z} 379$ peak measured on the

miniature LD-TOF-MS. (BOTTOM) The MS/MS spectrum

of the same peak measured on the commercial Bruker

MALDI TOF mass spectrometer. Clearly observed is the

same pattern of product ion peaks (highlighted).

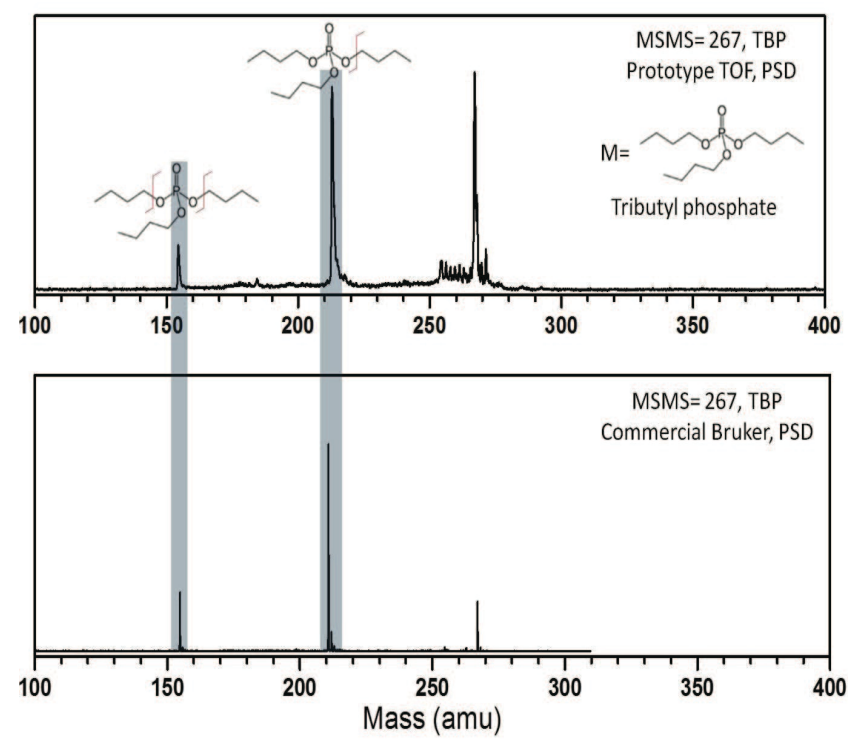


Figure 5. (TOP) MS/MS spectrum of protonated parent TBP ion, $\mathrm{m} / \mathrm{z} 267$ measured on miniaturized LD-TOF-MS. BOTTOM) MS/MS spectrum of the same peak measured on the Bruker MALDI TOF-MS. A similar pattern of the fragment peaks were observed (highlighted).

three equally spaced peaks in each spectrum corresponding to the consecutive losses of the side chains. Again, the PSD spectrum is found to be nearly identical to the CID product ion spectrum. ine: Among the 20 biological amino acids, valine is one that is also found in meteorites, and is thus a target molecules for studies of pre-biotic processes in the solar system. The MS/MS spectra of the gate-selected peak, protonated monomer of valine $(\mathrm{m} / \mathrm{z} 118)$ measured by miniaturized TOF-MS and the commercial instrument are shown in Figure 6 . The results on both instruments are quite consistent. The major fragment peak can be assigned as the loss of the - $\mathrm{COOH}$ group.

Dipeptides: Two dipeptides, glycine-glycine and aspartame, were also compared in the two mass spectrometers. Glycineglycine is the simplest peptide, and is useful as a buffer for many biological systems. Aspartame is a methyl ester of the aspartic acid-phenylalanine dipeptide that is used as an artificial sweetener. The MS/MS spectra of selected peaks are shown (Figs. 7 and 8). Here, the protonated monomer of glycine-glycine $(\mathrm{m} / \mathrm{z} 133)$, and protonated monomer of aspartame ( $\mathrm{m} / \mathrm{z} 295)$, were selected and measured. The results on both instruments are consistent for each dipeptide. Major fragment peaks can be assigned as the loss of various functional groups.
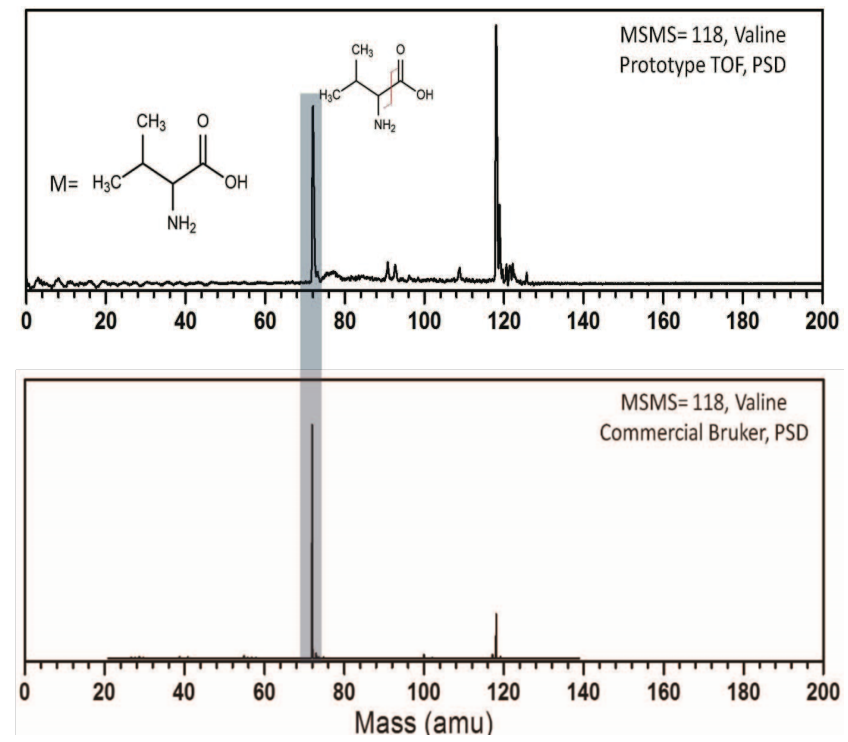

Figure 6. (TOP) MS/MS spectrum of protonated valine $(\mathrm{m} / \mathrm{z}$ 118) measured on miniaturized LD-TOF-MS. (BOTTOM) MS/MS spectrum of the same peak measured on the commercial Bruker MALDI TOF mass spectrometer. The same pattern of fragmentation peaks
Rhodamine 6G: Rhodamine $6 \mathrm{G}$ is a highly fluorescent dye. In our current LD-TOF-MS experiment, rhodamine $6 \mathrm{G}$ was used to demonstrate L-CID performance. The MS/MS spectrum of the main peak of the rhodamine $6 \mathrm{G}$ spectrum $(\mathrm{m} / \mathrm{z}=433)$, was measured under both PSD and L-CID modes (Figure 8). In PSD mode, there were several fragment peaks observed, but when used in the L-CID mode, a series of fragment ions at lower mass are observed indicating collisions with the desorbed gas provides sufficient energy for the parent ion to fragment into smaller species. Compared to data acquired on the commercial instrument (Figure 9), the same results were observed when a traditional CID gas (argon) was used. This provides evidence that L-CID and traditional CID exhibit similar collision energies and detection efficiencies.

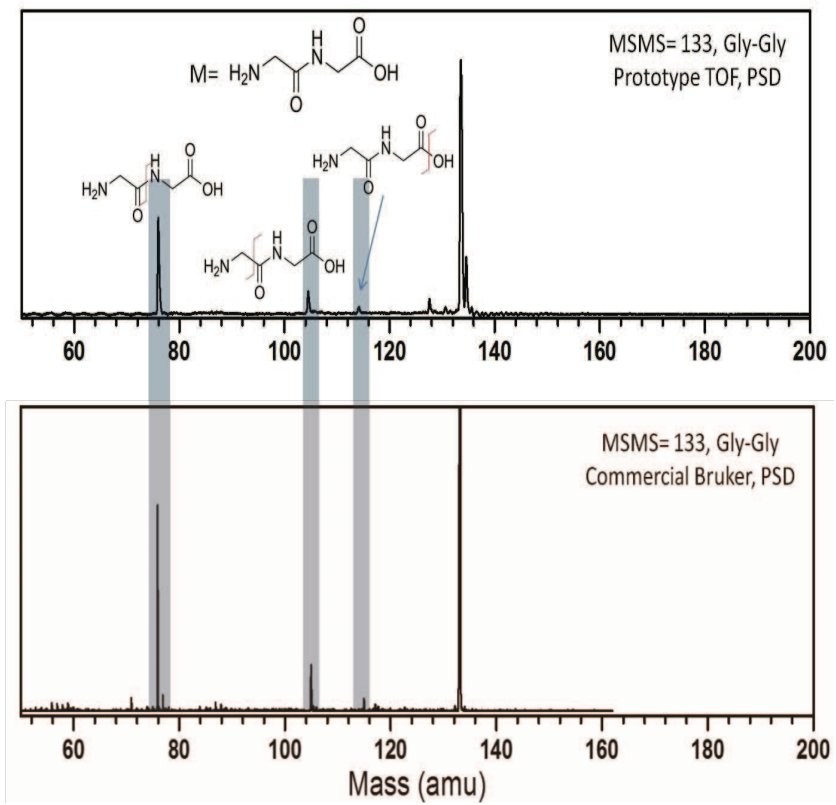

Figure 7. (TOP) The MS/MS spectrum of protonated parent glycine-glycine ion $(\mathrm{m} / \mathrm{z}=133)$ measured on the miniaturized TOF-MS. (BOTTOM)The MS/MS spectrum of the same peak measured on the commercial Bruker MALDI TOF mass spectrometer. 


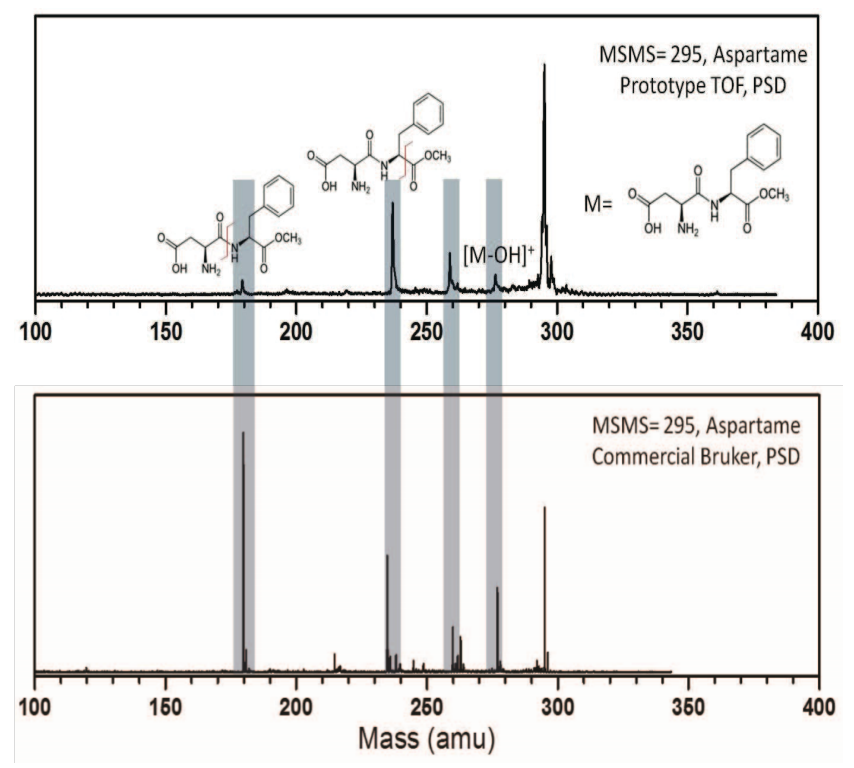

Figure 8. (TOP) The MS/MS spectrum of protonated parent aspartame ion, $(\mathrm{m} / \mathrm{z}=295)$ measured on miniaturized TOFMS. (BOTTOM)The MS/MS spectrum of the same peak measured on the commercial Bruker MALDI TOF mass spectrometer.

\section{DISCUSSION}

We demonstrated here the capability of performing tandem mass spectrometry on a miniaturized TOF-MS. It is noteworthy that systematic acquisition of PSD product ion data yields MS/MS spectra without adding any significant weight or volume to the instrument. Furthermore, it is remarkable that for many samples of interest, PSD efficiency is as high as that for CID (and this was found true even on the commercial instrument). In a real-world sample analysis scenario, most of the MS/MS measurements would require only PSD, while only specific compounds require L-CID.

The LDI technique advances the ability to desorb ions directly from a sample substrate to detect a range of nonvolatile and generally high molecular weight organics, as well as elemental abundances in complex samples. Coupling LDI to a highlycompact tandem TOF-MS then realizes a versatile tool for missions to explore Mars, Titan, the moons of Jupiter, Saturn and airless bodies (comets, asteroids and the Moon). Additionally, the MS/MS technique brings the extremely important molecular structure analysis capability to bear on individual organic molecules, a key step on the NASA Roadmap, which seeks to understand the origin and history of the Solar System and the potential for life elsewhere. Carefully-planned MS/MS application to complex unknowns can help resolve the hundreds (or more) possible candidates for a given mass peak, particulary when examining potential molecular biosignatures for which the detailed structure (e.g., side groups) are most telling of function.

\section{OUTLOOK}

The miniaturized LD-TOF-MS, including MS/MS capability, offers a rapid, low cost, portable technology for the detection and structural analysis of semi-volatile and nonvolatile microorganisms, organics, trace metals, elemental abundances as well as in Earth-bound applications such as detection of explosives and food pathogens. To further improve the instrument, we will study the MS/MS measurement more in the negative ion mode. Although the instrument is capable of rapidly switching between symmetric detection of ions with either positive or negative polarities, the efficiency of the negative ions PSD compare to positive ions is not well-characterized at this point. Furthermore, an important next goal will be to develop the full tandem LD-TOF-MS at flight scale to a technology readiness level (TRL) from its current level of 5 up to 6 , indicating compliance with the launch, spaceflight, and operational environments and readiness for flight development. 


\section{REFERENCES}

[1] Johnson PV, Beegle LW, and Kanik I. Mass Spectrometry in Solar System Exploration. Mass Spectrometry Handbook: John Wiley \& Sons, Inc.; 2012. p. 389-405.

[2] Arevalo $R$, Brinckerhoff $W$, van Amerom $F$, Danell $R$, Pinnick V, Xiang L, Getty S, Hovmand L, Grubisic A, Mahaffy P, Goesmann F, and Steininger $H$, editors. Design and demonstration of the Mars Organic Molecule Analyzer (MOMA) on the ExoMars 2018 rover. Aerospace Conference, 2015 IEEE; 2015 7-14 March 2015.

[3] Brinckerhoff WB, Pinnick VT, van Amerom FHW, Danell RM, Arevalo RD, Atanassova MS, Xiang L, Mahaffy PR, Cotter RJ, Goesmann F, and Steininger $\mathrm{H}$, editors. Mars Organic Molecule Analyzer (MOMA) mass spectrometer
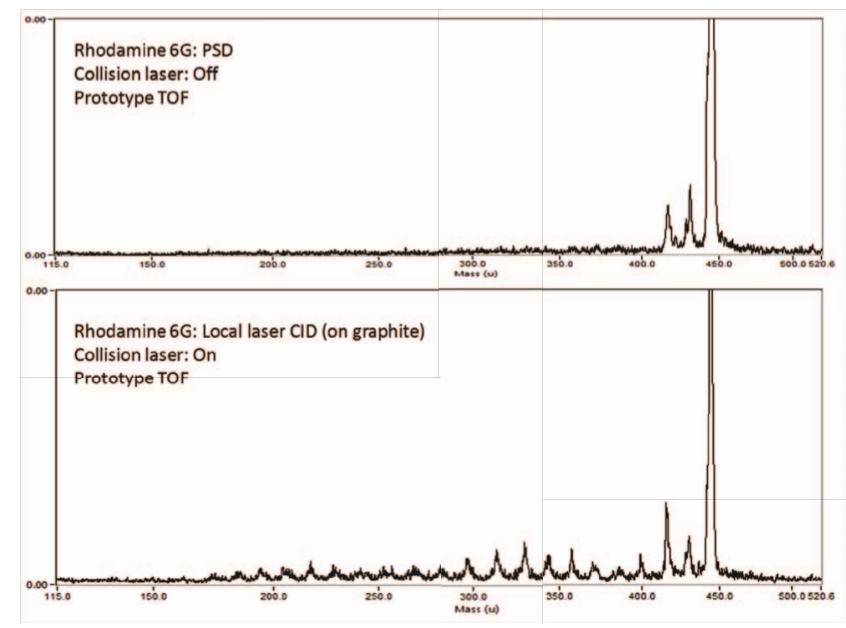

[6] Cornish TJ, and Cotter RJ. "A curved-field reflectron for improved energy focusing of product ions in time-of-flight mass spectrometry". Rapid Communications in Mass Spectrometry.7:1037-40; (1993).

[7] Cornish TJ, Ecelberger S, and Brinckerhoff W. "Miniature time-of-flight mass spectrometer using a flexible circuitboard reflector". Rapid Communications in Mass Spectrometry.14:2408-11; (2000).

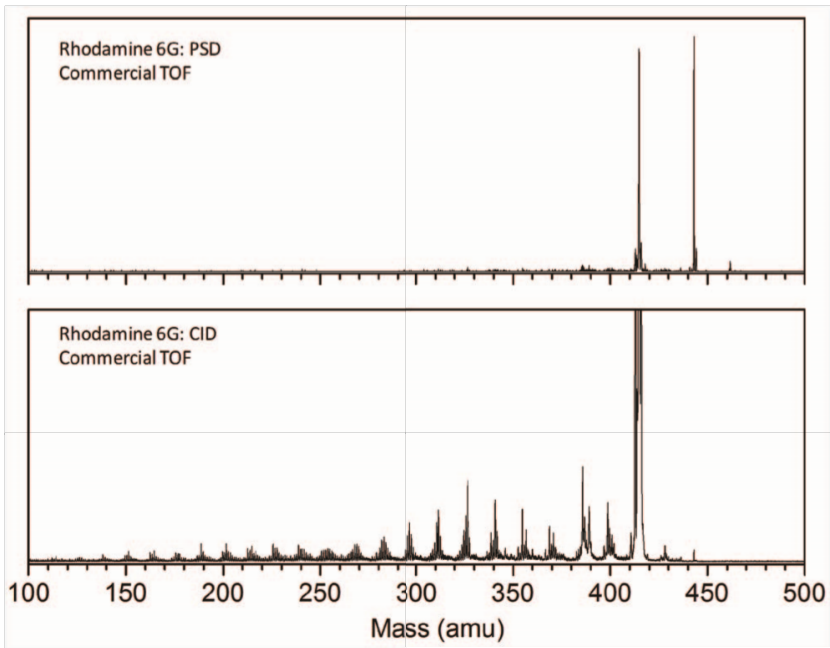

Figure 9. (LEFT) The MS/MS spectrum of rhodamine $6 \mathrm{G}, \mathrm{m}=443$, measured on miniaturized TOF-MS with PSD and L-CID. (RIGHT) The MS/MS spectrum of rhodamine 6G, $m=443$, measured on the commercial TOF mass spectrometer with PSD and CID. Same pattern of the fragment peaks were observed.

for ExoMars 2018 and beyond. Aerospace Conference, 2013 IEEE; 2013 2-9 March 2013.

[4] Getty SA, Brinckerhoff WB, Cornish T, Ecelberger S, and Floyd M. "Compact two-step laser time-of-flight mass spectrometer for in situ analyses of aromatic organics on planetary missions". Rapid Communications in Mass Spectrometry.26:2786-90; (2012).

\section{BIOGRAPHY}

[5] Suckau D, Resemann A, Schuerenberg M, Hufnagel $P$, Franzen J, and Holle A. "A novel MALDI LIFT-TOF/TOF mass spectrometer for proteomics". Anal Bioanal Chem.376:952-65; (2003).

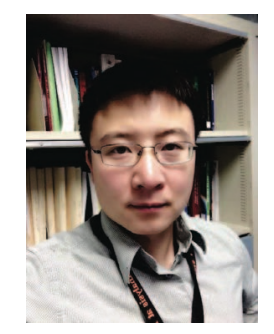

Xiang $L i$ received his B.S. in Chemistry from the Peking University, China in 2003, and Ph.D. in Physical Chemistry from the Johns Hopkins University in 2009. He is an assistant research 
scientist with a joint appointment at the University of Maryland, Baltimore County and NASA Goddard Space Flight

Center. His research focuses on the

detection of trace element and astrobiologically relevant organic molecules in planetary systems, like Mars. He is especially interested in the instrument development of timeof-flight and ion trap mass spectrometers with various ionization and ion gating techniques. He serves as mass spec. scientist for the MOMA ion trap MS on ExoMars and Co-I on development of LITMS and MACROS instrument.

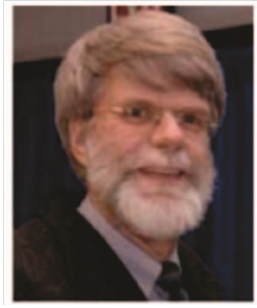

Timothy Cornish Ph.D., President of C\&E Research, Inc. in Columbia, MD, a scientific consulting and CRAD company specializing in miniature Time Of Flight Mass Spectrometry from conceptual design to prototype $d$ evelopment and testing. He has extensive experience in high resolution optical spectroscopy and laser applications for chemical analysis. His recent efforts have focused on miniature mass spectrometry with targeted developments directed toward both mineralogical and biomarker analysis on landed planetary missions, as well as development of a high performance tandem mass spectrometry for field-portable terrestrial applications.

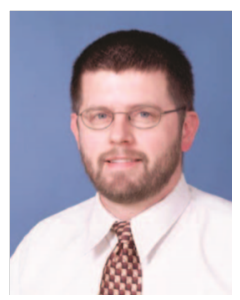

Scott Ecelberger is Vice President and cofounder of C\&E Research, Inc. in Columbia MD. He has an M.S. in Applied Physics from the Johns Hopkins University and a B.S. in Physics from Penn State. Scott is a hands-on experimental physicist who designs, builds, and tests analytical instrumentation. He

has broad experience with vacuum systems, lasers and optics, high speed and high voltage electronics, BSL-3 containment labs, vacuum deposition systems, semiconductor characterization, and TOF Mass Spectrometry. Current projects include miniature TOF mass spectrometry for mineralogy and bio-defense and single particle mass spec instrumentation for aerosol analysis.

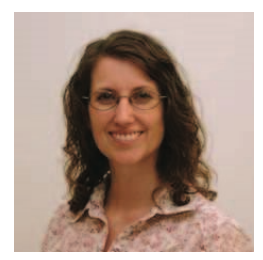

Stephanie Getty is a Research Planetary Scientist in the Planetary Environments Laboratory at NASA's Goddard Space Flight Center with interests in the development of advanced instrumentation for the compositional analysis of planetary environments. She currently serves as Principal Investigator of the PIDD-funded development of a two-step laser tandem time-of-flight mass spectrometer for targeted elucidation of organics in non-volatile planetary surface chemistry. She also serves as Principal Investigator of the ASTID-funded development of OASIS, a liquid chromatography-mass spectrometer for in situ analysis of prebiotic compounds. She is a member of the ExoMars MOMA-MS team and a Mars Science Laboratory Collaborator.

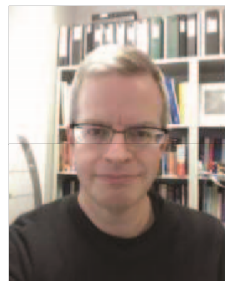

William Brinckerhoff is a senior space research associate at NASA's Goddard Space Flight Center. He received his Ph.D. in Physics from the Ohio State University. His current research interests include development of novel miniature mass spectrometers and sample handling systems for planetary missions, synthesis of organic compounds in the interstellar medium, and the habitability of Mars. He serves as a Co-I on MSL/SAM and project scientist for the MOMA ion trap MS on ExoMars. 